\title{
A Importância da Linguagem Nativa para a Aprendizagem Significativa em Lógica de Programação
}

\author{
Geovana R. S. Silva ${ }^{1}$, Giovanni A. Santos ${ }^{2}$, Vandor R. V. Rissoli ${ }^{1}$ \\ ${ }^{1}$ Laboratóro de Tecnologias Educacionais (LaTEd) - Faculdade do Gama (FGA) \\ Universidade de Brasília (UnB), Brasília, DF \\ ${ }^{2}$ Departamento de Engenharia Elétrica \\ Universidade de Brasília (UnB), Brasília, DF \\ geovana.ramosealuno.unb.br, \{giovannix, vandor\}@unb.br
}

\begin{abstract}
The initial learning of algorithms becomes harder when the programming language used has keywords in a foreign language not known by the student. The purpose of this study is to analyze the use of the learner's native language when starting to learn to program. A questionnaire was applied at the end of four consecutive semesters of an introductory programming course, and five questions were selected to identify the level of student satisfaction and the efficiency of the educational process. The results obtained show that most of the students interviewed were satisfied, had their expectations met and recommended the methodology used.
\end{abstract}

Resumo. O aprendizado inicial de algoritmos se torna mais árduo quando a linguagem de programação utilizada possui comandos em uma língua estrangeira não dominada pelo aluno. O propósito deste estudo é analisar o uso da linguagem nativa do aprendiz nos estudos iniciais de programação. Um questionário foi aplicado ao final de quatro semestres consecutivos de um curso introdutório de programação e cinco perguntas foram selecionadas para identificar o nível de satisfação dos alunos e a eficiência do processo educacional. Os resultados obtidos mostram que a maior parte dos alunos entrevistados se mostrou satisfeita, teve as suas expectativas atendidas e recomenda a metodologia utilizada.

\section{Introdução}

Aprender uma linguagem de programação pela primeira vez envolve o desenvolvimento de um conjunto de habilidades. Além das dificuldades mais comuns, os alunos que não têm o inglês como língua nativa também precisam lidar com o desconhecimento de palavras-chave e instruções da linguagem de programação que, geralmente, estão em inglês [Gu et al, 2017]. Conforme detalhado por Watson e Li (2014), cuja pesquisa analisou dados de disciplinas de lógica de programação ofertadas no período de 1979 a 2013, os países que apresentaram as maiores taxas médias de insucesso dos alunos foram Portugal, Alemanha e Brasil, justamente, países que não têm o inglês como idioma nativo.

Por isso, muitos educadores recorrem a alternativas que busquem abstrair as complexidades das linguagens de programação, como o uso de pseudolinguagens. Diante do desafio dessa nova aprendizagem, a relevância de aspectos relacionados aos fatores metodológicos é fundamental para o seu êxito, principalmente, quando se almeja que essa aprendizagem seja significativa para os estudantes. Ausubel (2000) esclarece que tal situação é obtida com maior facilidade quando se promove a associação do conhecimento estabelecido 
nas estruturas cognitivas dos aprendizes com o novo conteúdo. Dessa forma, o novo vai se tornando significativo ao aprendiz que o assimila de forma mais natural e segura, pois o integra aquilo que ele já sabe.

Esse processo de associação, denominado de subsunção, reconhece a relevância de um entendimento inicial sobre o que se deseja apresentar de novo para os aprendizes, que naturalmente possuem algum domínio sobre a sua linguagem nativa de comunicação. Quando essa não pode ser usada amplamente na assimilação de algo novo, a subsunção é comprometida e demandará de mais esforço do estudante que almeja aprender.

Diante do desafio do processo de ensino-aprendizagem de programação e à luz da aprendizagem significativa, esta pesquisa melhor compreende o impacto do uso de uma linguagem de programação com instruções em pseudocódigo escritas na linguagem nativa do aprendiz. O ambiente de software utilizado é o Calango, o qual possui uma interface gráfica que permite a depuração do código e funcionalidades que oferecem facilidades aos alunos iniciantes na área.

Este artigo está organizado em mais seis seções. A seção 2 apresenta aspectos que contextualizam esta pesquisa. A seção 3 mostra detalhes do ambiente de desenvolvimento Calango, enquanto a seção 4 esclarece aspectos principais da metodologia utilizada. A seção 5 apresenta os resultados observados, estando as suas conclusões na seção 6 .

\section{Contextualização}

Tradicionalmente, o início na área da programação é difícil, podendo ser constatado pela taxa média de reprovação mundial estar em cerca de 30\% [Watson e Li, 2014]. No Brasil esta taxa está acima do nível mundial, sendo superior as taxas de outros cursos de bacharelado [Bosse e Gerosa, 2015]. Um dos campos de investigação relacionados ao aprendizado inicial em programação busca motivar os alunos no processo de aprendizagem envolvendo diferentes metodologias.

Entre as alternativas, o uso de pseudocódigo tem se destacado no ensino introdutório à programação. A expressão pseudocódigo em programação indica a elaboração do esboço de um programa que usa uma linguagem menos rígida e mais condizente com a comunicação dos envolvidos, podendo mais facilmente ser transformado em instruções pertencentes a uma linguagem de programação em momento posterior [Karatrantou et al, 2008]. O pseudocódigo propicia uma escrita mais próxima a linguagem natural, a qual o aprendiz tem mais domínio.

Devido a esse fato, Olsen (2005) mudou a sequência dos itens lecionados no ensino de programação, começando primeiro com pseudocódigo, em vez de iniciar com a linguagem $\mathrm{C}++$. Embora os resultados tenham sido positivos, os alunos não possuíam uma ferramenta capaz de executar os pseudocódigos elaborados, comprometendo o entendimento dos aprendizes de como os programas de computador funcionam e implicando na dificuldade da transição do pseudocódigo para a linguagem $\mathrm{C}++$.

Ezenwoye (2018) observa em seu trabalho que as disciplinas iniciais em lógica de programação empregam, geralmente, linguagens em inglês, enquanto Guo (2018) constatou que os estudantes que têm o português como língua nativa, possuem muitas dificuldades relacionadas à compreensão das palavras-chave e comandos em inglês. Na pesquisa liberada de Olatunji et al (2019), 89\% dos entrevistados informam que gostariam de programar em seu idioma nativo, reforçando a importância de buscar associar o que se sabe com o novo. 
Em Oliveira, Monteiro e Roman (2013), são usadas duas linguagens de programação desenvolvidas para serem mais próximas à linguagem de comunicação nativa. Uma dessas linguagens era muito similar ao português natural e a outra seguia uma notação gramatical livre de contexto clássico, com aparência de pseudocódigo. Porém, as linguagens são apenas utilizadas em um jogo, o que limita seu uso.

As posturas educacionais e as ferramentas ou ambientes analisados se mostraram eficientes em seus objetivos, mas em uma abordagem mais ampla, nenhum se destacou como uma solução completa. Essa realidade motivou a busca de alternativas de suporte tecnológico à postura educacional baseada em alguns princípios proposto pela Teoria da Aprendizagem Significativa (TAS), em que a subsunção, apoiada pela linguagem nativa dos aprendizes, corresponde a um dos aspectos mais relevantes ao sucesso do processo educacional inicial em lógica de programação.

Dessa forma, foi desenvolvido o Ambiente de Desenvolvimento Integrado Calango, que faz uso da língua portuguesa na construção de pseudocódigos que são empregados na elaboração de algoritmos capazes de solucionarem problemas de programação em um ambiente gráfico.

\section{Ambiente de Desenvolvimento Integrado Calango}

O Calango é um software educacional multiplataforma que permite a construção, depuração e manutenção de algoritmos, escritos em pseudocódigo na língua portuguesa. Por meio dela os aprendizes elaboram soluções e o computador as executa como se fossem programas elaborados em uma linguagem de programação, facilitando a compreensão de como um programa realmente executaria. A sua utilização como recurso de apoio à aprendizagem inicial em programação de computadores almeja ainda:

- Maior facilidade na aprendizagem de programação aos nativos em língua portuguesa;

- Uso de representação mais simples em algoritmo que pode solucionar o problema proposto usando a linguagem que o estudante domina;

- Execução do algoritmo pelo computador com orientações na língua do aprendiz;

- Depuração do pseudocódigo em tempo real, sendo executado passo a passo e apresentando ao aprendiz a realidade momentânea de cada valor armazenado na memória, similar ao teste de mesa em programação;

- Autonomia para os estudantes novatos em programação, possibilitando um processo de aprendizagem continuada e significativa, que considera o ritmo individual de cada aprendiz;

- Assistência na depuração do pseudocódigo, melhor averiguando possíveis erros ou depurando o algoritmo para aprimorá-lo.

O pseudocódigo usado pelo Calango foi baseado nas características estruturais da linguagem $\mathrm{C}$, por esta ser notoriamente reconhecida e amplamente usada ao introduzir o ensino da programação [Ezenwoye, 2018]. Além dos operadores e comentários análogos aos disponíveis na linguagem $\mathrm{C}$, o Calango ainda trabalha com uma notação mais instrutiva para a marcação de blocos de processamento.

\section{Metodologia}

Com uma postura ativa e empenhado em modificar a situação educacional estabelecida no ensino dos conteúdos introdutórios em programação computacional, um grupo de docentes 
participou de um curso de extensão universitária antes do experimento. Este curso abordava, principalmente, questões metodológicas da TAS associadas a uma postura mais ativa e preocupada com a aprendizagem discente.

A disciplina escolhida para utilizar o Calango na instituição lecionou a linguagem C por muitos anos e é ministrada para estudantes de diferentes engenharias. Durante quatro semestres consecutivos o Calango foi apresentado antes da linguagem $\mathrm{C}$ e no final do período letivo, após a realização de todas as atividades avaliativas da disciplina, todos os alunos eram convidados a participarem de uma pesquisa que solicitava as suas opiniões sobre a metodologia de ensino e os recursos utilizados. Cinco questões dessa pesquisa foram selecionadas para contribuir com as análises referentes ao uso do Calango, sendo complementar as considerações possíveis em cada uma delas.

\subsection{Experimento da Pesquisa}

Os alunos que participaram do experimento proposto são graduandos da Universidade de Brasília (UnB), que oferece cursos de bacharelado em engenharias (Eletrônica, de Energia, Automotiva, Aeroespacial e de Software). Todos os alunos desses cursos precisam fazer a disciplina incial em lógica de programação, denominada Algoritmos e Programação de Computadores (APC).

Uma pesquisa de opinião do tipo Survey foi aplicada ao fim de quatro períodos letivos (semestres) consecutivos envolvendo o mesmo docente na condução dessas turmas (uma por semestre). Com isso, essa pesquisa obteve uma amostragem aleatório simples envolvendo 264 alunos convidados a participarem, respondendo a um questionário virtual e sigiloso (sem identificação do respondente).

Esse instrumento de pesquisa foi preparado por uma equipe multidisciplinar de docentes das áreas de Educação, Informática, Matemática e Psicologia. Foi inspirado pela Students' Evaluation of Educational Quality (SEEQ) e buscava averiguar o nível de satisfação dos alunos e a eficiência do processo educacional [Watkins et al, 1987]. Essa pesquisa era composta por 19 questões, abordando cinco assuntos a partir da perspectiva de cada aluno participante: $i$ ) características pessoais do participante; $i$ i) recursos tecnológicos usados no apoio educacional; iii) postura pedagógica docente; iv) monitoria estudantil; v) análise pessoal.

Todos os estudantes matriculados na disciplina foram convidados a participar, mas nem todos os que a iniciaram responderam à pesquisa, pois é possível desistir da inscrição até um determinado período adiantado do semestre letivo que é definido pela instituição. Portanto, o número de alunos indicados nesta pesquisa corresponde a quantidade de estudantes que concluíram a disciplina, com ou sem sucesso. Além disso, nem todas as perguntas foram respondidas por todos os participantes, pois algumas não eram obrigatórias ou dependiam da resposta de uma questão anterior.

Embora APC seja oferecida como presencial, algumas das ferramentas tecnológicas adotadas fornecem suporte ao processo de ensino a distância, permitindo o acompanhamento personalizado da situação de aprendizagem. Uma delas é o Sistema Tutor Inteligente conhecido como SAE (Sistema de Apoio Educacional) que fornece suporte interativo e mais realista sobre a situação atual da aprendizagem individual dos estudantes, analisando o desempenho e o esforço de cada aprendiz sobre cada um dos conteúdos ou conceitos importantes que compõem a disciplina. 
O SAE emprega a Lógica Fuzzy para inferir essa situação de aprendizagem individual como "Satisfatória" ou "Insatisfatória" à aprendizagem ser significativa à estrutura cognitiva do aprendiz [Rissoli, 2007]. Ao final da disciplina, se o aprendiz alcançar a situação "Satisfatória" em todos os conteúdos estudados, lhe será atribuído um índice que corresponde a uma ponderação envolvendo os valores de confiança (ou graus de pertinência em cada conteúdo ou conceito da disciplina). O cálculo do grau do disparo sobre o conjunto fuzzy reflete a situação de aprendizagem mais realista dos alunos em cada conteúdo disciplinar [Klir e Yuan, 1995].

Além das notas, geralmente alcançadas em atividades avaliativas pré-agendadas com as turmas no decorrer do período letivo, o cálculo desse índice significativo também é importante, pois é obtido pelo sistema que considera vários aspectos relacionados ao aprendizado dos alunos em cada conteúdo da disciplina, o que raramente as notas conseguem indicar. Entre estes aspectos se incluem a quantidade e o resultado das perguntas respondidas por item do conteúdo, participação em atividades indicadas pelo professor, além da frequência na monitoria da disciplina em período extraclasse. Para a disciplina escolhida nesta pesquisa, o índice significativo varia de 0 a 10 e um resultado considerado satisfatório deve estar acima de 5.

Como o objetivo desta pesquisa não é ser comprometida por posturas educacionais diferentes, foram analisadas somente as turmas de APC conduzidas pelo mesmo docente, que aplicou o mesmo plano de ensino nas quatro turmas analisadas. A postura pedagógica do professor foi baseada na proposta da TAS, que reconhece a importância da associação entre o conhecimento estabelecido na estrutura cognitiva do aprendiz e o novo conteúdo que lhe é apresentado [Ausubel et al, 1968].

\subsection{Instrumento da Pesquisa de Opinião}

$\mathrm{O}$ instrumento de pesquisa aplicado nas turmas foi interativo e virtual, protegido por senha única associada a cada questionário, mas sem a identificação dos respondentes. Elaborado pelo software de código aberto denominado Lime Survey ${ }^{l}$, cinco questões foram selecionadas para análise nesta pesquisa, pois abordavam aspectos relativos a metodologia educacional em diferentes perspectivas, podendo mostrar aspectos diferentes no impacto do estudo inicial em lógica de programação com o Calango.

Q.1 Você diria que o professor está usando alguma metodologia de aprendizagem ou nenhum método estaria sendo usado por ele na condução da disciplina?

Q.2 Informe o valor de seu índice significativo atribuído pelo SAE?

Q.3 As ferramentas de desenvolvimento de código adotadas por esta disciplina foram importantes para o seu aprendizado? Quais foram suas impressões sobre aprender usando o Calango antes da Linguagem C?

Q.4 Na sua opinião, APC atendeu suas expectativas iniciais no semestre letivo?

Q.5 Você recomendaria a seus colegas cursarem esta disciplina como você fez, nessa forma de ensino e usando esses recursos de suporte tecnológico?

\footnotetext{
${ }^{1}$ https://www.limesurvey.org/pt/LimeSurvey
} 
As demais questões do instrumento procuravam identificar melhor o perfil pessoal do aluno e as experiências pregressas usando tecnologias e recursos de softwares, a princípio não relevantes aos objetivos dessa pesquisa.

\section{Resultados}

A inquietude do docente em buscar alternativas para a mudança da realidade constatada a partir de metodologias educacionais coerentes corresponde a um resultado relevante nesta pesquisa e demonstra o reconhecimento da importância da aprendizagem ser significativa aos aprendizes iniciantes em programação.

A andragogia estabelecida pelo professor com as turmas era mais consciente no uso de materiais instrucionais que fossem potencialmente mais significativos aos estudantes, enquanto o docente procurava mediar o processo educacional e motivar cada estudante a aplicar aquilo que já sabia na compreensão do novo (subsunção).

Na primeira questão selecionada do instrumento (Q.1) é possível notar a percepção dos alunos quanto ao esforço docente em procurar facilitar o aprendizado de cada estudante. O resultado obtido nesta questão objetiva está indicado na Tabela 1.

Nas 182 respostas fornecidas, 94\% afirmavam, explicitamente, que uma metodologia foi utilizada, podendo este valor ser considerado um indicador de quão bem a disciplina foi lecionada e que o docente seguiu aspectos metodológicos para acompanhar e avaliar mais a percepção dos alunos sobre o uso do Calango.

Tabela 1. Estudantes que perceberam o uso de uma metodologia de aprendizagem.

\begin{tabular}{|c|c|c|c|c|c|}
\hline Ano Letivo & Semestre & Respostas & SIM & NÃO & NÃO SEI DIZER \\
\hline 2018 & 1 & 31 & 28 & 1 & 2 \\
\hline 2018 & 2 & 28 & 28 & 0 & 0 \\
\hline 2019 & 1 & 66 & 62 & 1 & 3 \\
\hline 2019 & 2 & 57 & 53 & 0 & 4 \\
\hline \multicolumn{2}{|c|}{ Total } & $\mathbf{1 8 2}$ & $\mathbf{1 7 1}$ & $\mathbf{2}$ & $\mathbf{9}$ \\
\hline
\end{tabular}

A Q.2 indica a eficiência do processo educacional considerando a situação de aprendizagem atingida pelos estudantes em cada conteúdo que compõe a disciplina. $\mathrm{O}$ fornecimento do índice significativo pelo SAE somente ocorre quando os aprendizes atingem a classificação satisfatória de aprendizagem em cada um desses conteúdos. Assim, os estudantes que forneceram o valor do índice na Q.2 alcançaram esta situação.

Tabela 2a. Alunos que concluíram a disciplina.

\begin{tabular}{|c|c|c|}
\hline Período Letivo & SIM & NÃO \\
\hline $2018 / 1$ & 09 & 14 \\
\hline $2018 / 2$ & 11 & 13 \\
\hline $2019 / 1$ & 44 & 07 \\
\hline $2019 / 2$ & 26 & 14 \\
\hline Total & $\mathbf{9 0}$ & $\mathbf{4 8}$ \\
\hline
\end{tabular}

Tabela 2b. Índices alcançados pelos alunos concluintes

\begin{tabular}{|c|c|c|}
\hline Período Letivo & Respostas & Média \\
\hline $2018 / 1$ & 09 & 6,72 \\
\hline $2018 / 2$ & 11 & 5,98 \\
\hline $2019 / 1$ & 43 & 6,77 \\
\hline $2019 / 2$ & 25 & 6,76 \\
\hline Total & $\mathbf{8 8}$ & $\mathbf{6 , 5 6}$ \\
\hline
\end{tabular}

A Tabela 2a mostra os estudantes que participaram da pesquisa e concluíram a disciplina, enquanto que a Tabela $2 \mathrm{~b}$ apresenta a média aritmética obtida pelos estudantes de 
cada turma no índice significativo. Na totalização destas duas tabelas é possível observar que dos 90 concluintes 88 informaram o índice significativo $(97,7 \%)$, pois não era uma questão obrigatória. Considerando que a atribuição formal de notas em APC varia de 0 a 10 , que a nota final mínima para aprovação na instituição é 5, e que o índice significativo fornecido pelo SAE analisa uma amplitude maior de fatores que interferem no processo educacional, empregando Fuzzy para mensurar a realidade da situação avaliada, foram obtidos resultados significativos das turmas analisadas (média geral do índice superior a nota de aprovação $6,56)$.

A Q.3 propicia uma análise que vai além de saber se os discentes gostaram ou não do uso do Calango em APC, pois solicitava uma justificativa livre aos estudantes que participaram da pesquisa. Assim, torna-se possível melhor conhecer o porquê da opinião de cada um, que ainda fundamentava a sua experiência e seu ponto de vista.

Inicialmente, todas as respostas recebidas na Q.3 foram lidas sem qualquer classificação, a fim de se analisar quais eram as impressões gerais dos alunos participantes. Depois que as declarações mais comuns foram identificadas, outra rodada de leitura foi realizada com o objetivo de contar quantos alunos citavam essas impressões comuns. Para evitar interpretações errôneas, apenas as respostas que expressavam, explicitamente, uma declaração ou opinião foram consideradas nessa contagem. Além disso, como essa questão também compreendia as outras ferramentas usadas na disciplina, apenas foram contadas as que citavam expressamente o Calango.

Dessa forma, foram contabilizadas 184 respostas, das quais 147 abordaram explicitamente o Calango. Aproximadamente $83 \%$ das respostas sobre o Calango foram positivas e na maioria delas os estudantes informavam que o Calango reforçou o pensamento lógico e que a transição para a linguagem $\mathrm{C}$ não foi difícil devido ao estudo inicial tê-lo utilizado. Dentre as diversas opiniões coletadas duas declarações de estudantes diferentes são apresentadas como exemplo:

"A ferramenta do Calango foi uma das mais importantes, pois introduziu a lógica necessária na matéria e facilitou muito no uso da linguagem C por ser muito similar."

"Ter aprendido primeiro a lógica através do Calango foi algo extremamente útil. Depois que se aprende a lógica é muito mais fácil programar em outras linguagens. Ao comparar o desempenho da minha turma com o de outras, percebi a dificuldade que meus colegas de outras turmas enfrentavam ao fazer códigos que minha turma já tinha feito a mais de um mês."

Vários outros depoimentos ressaltavam a importância do estudo da lógica de programação com o Calango para aqueles que nunca tinham tido contato com a programação, além da relevância do uso da língua nativa como fator facilitador na assimilação do conteúdo. Dois depoimentos de alunos diferentes são compartilhados como exemplos:

"Acredito que aprender a lógica de programação, começando em português (com Calango) para depois ir pra Linguagem $C$ é indispensável pra quem nunca teve contanto com qualquer linguagem (como eu)."

"O Calango sem dúvida ajuda bastante introduzindo a lógica de programação, pra quem nunca teve contado é de grande importância se familiarizar com os termos primeiro em português e depois trabalha-los em inglês." 
Independente da aprovação ou não dos estudantes em APC, a Q.4 questionava todos os alunos se as suas próprias expectativas haviam sido atendidas e $76 \%$ afirmavam que sim, indicando que estavam satisfeitos com a disciplina. Como pode ser observado na Tabela 3 , se a análise sobre Q.4 separar os discentes satisfeitos e os que tiveram as expectativas atendidas os resultados finais são ainda mais positivos.

Tabela 3. 0 sentimento de satisfação dos estudantes ao final da disciplina.

\begin{tabular}{|c|c|c|c|c|c|}
\hline Ano Letivo & \multicolumn{2}{|c|}{2018} & \multicolumn{2}{|c|}{2019} & Total \\
\hline Semestre & 1 & 2 & 1 & 2 & Geral \\
\hline Sim, e estou satisfeito & 16 & 22 & 54 & 47 & 139 \\
\hline Sim, mas estou insatisfeito & 05 & 01 & 05 & 06 & 17 \\
\hline Não, e estou insatisfeito & 05 & 01 & 03 & 01 & 10 \\
\hline Não, mas estou satisfeito & 05 & 04 & 04 & 03 & 16 \\
\hline Total & 31 & 28 & 66 & 57 & 182 \\
\hline
\end{tabular}

Como Q.4 era objetiva, com quatro opções de resposta, o nível de expectativas atendidas alcança $86 \%$, enquanto a satisfação com a disciplina chega a $85 \%$, sendo analisadas separadamente. Estes valores sinalizam o alinhamento do conteúdo proposto pelo docente e o desejo dos aprendizes em APC, além de quantificar o nível de satisfação dos alunos com a metodologia empregada usando o Calango.

A Q.5 é objetiva e solicita de forma direta se o aluno que fez APC a recomendaria para outro colega cursá-la nos moldes metodológicos e tecnológicos que ele acabou de fazer. Das 182 respostas, 95\% afirmaram que a recomendariam. Um complemento dessa questão solicitava a justificativa do porquê fariam a recomendação ou não. Destacou-se a indicação de que o Calango deveria continuar sendo usado na disciplina. A Tabela 4 apresenta as respostas obtidas.

Tabela 4. Alunos que recomendariam esta disciplina aos colegas.

\begin{tabular}{|c|c|c|c|}
\hline Período Letivo & Respostas & SIM & NÃO \\
\hline $2018 / 1$ & 31 & 29 & 2 \\
\hline $2018 / 2$ & 28 & 28 & 0 \\
\hline $2019 / 1$ & 66 & 63 & 3 \\
\hline $2019 / 2$ & 57 & 53 & 4 \\
\hline Total & $\mathbf{1 8 2}$ & $\mathbf{1 7 3}$ & $\mathbf{9}$ \\
\hline
\end{tabular}

Os quatro semestres em conjunto tiveram uma média de 56,3\% de aprovação e 21,3\% de abandono. O percentual de abandono inclui alunos que realizaram o trancamento da disciplina ou não atingiram $75 \%$ de presença nas aulas. Desconsiderando os abandonos, a média de aprovação quando utilizando o Calango foi de 72,3\%.

Um outro aspecto que deve ser analisado é o índice de aprovação em semestres que não usaram o Calango na mesma disciplina e com o mesmo professor. Ao analisar dois semestres anteriores, que não utilizaram o Calango, a média de aprovação foi de $33 \%$ e de abandono foi de $28,6 \%$. Novamente, desconsiderando os abandonos, tem-se a média de $46,5 \%$ de aprovação. Ou seja, a média de aprovação subiu de 46,5\% em média para 72,3\% com a utilização do Calango.

Outro fato interessante de se observar é o aumento do percentual de alunos que alcançaram média final superior a sete, que foi de $9,2 \%$ para $29,13 \%$ com o uso do Calango. 
IX Congresso Brasileiro de Informática na Educação (CBIE 2020)

Anais do XXXI Simpósio Brasileiro de Informática na Educação (SBIE 2020)

Em conjunto com o aumento de aprovação, estes dados indicam a elevação no rendimento dos aprovados, sinalizando a melhoria na eficiência da aprendizagem na disciplina.

\section{Conclusões}

Este trabalho mostra a ação inicial partindo do docente, o qual está envolvido com os desafios do processo educacional em introdução a programação. Estando ele descontente com a realidade, se apoiou na Teoria da Aprendizagem Significativa e em novas tecnologias que poderiam fornecer apoio coerente à melhor andragogia na condução de suas turmas, além de trabalhar habilidades relevantes à formação de seus alunos.

Isso possibilitou a elaboração de um novo ambiente educacional, o qual permite maior autonomia dos aprendizes com aporte tecnológico coerente à aprendizagem significativa almejada. Com estudo mais interativo e envolvendo o Calango como ambiente fundamental na aprendizagem, os estudantes podiam organizar seus próprios horários de estudo extraclasse empregando uma ferramenta que interagia através da língua que o aprendiz dominava e fornecia orientação direta no nível em que ele se encontrava, similar aos ambientes de programação reais.

As respostas obtidas na pesquisa indicam aspectos do êxito educacional acompanhando o nível de assimilação individual dos estudantes, em que $95 \%$ dos alunos recomendaram que outros discentes cursassem essa disciplina. Também foi observado uma maior participação dos estudantes, inclusive pelos próprios alunos, que relataram nas suas autoavaliações um nível de participação elevado na disciplina ( $32 \%$ ótima e $60 \%$ boa). Com 92\% de maior participação é possível inferir que os aprendizes também estavam mais motivados.

A principal contribuição deste trabalho está na combinação da metodologia com os recursos tecnológicos que lhe sejam coerentes aos objetivos almejados, promovendo um ambiente mais autônomo e sintonizado com as características dos aprendizes, a fim de fomentar um processo educacional mais voltado à aprendizagem que seja significativa e amplie seus conhecimentos de forma mais natural, usufruindo da língua que o aprendiz dominada para o êxito da subsunção necessária ao sucesso do processo educacional em Programação Computacional.

\section{Referências}

Ausubel, D. (2000) The Acquisition and Retention of Knowledge: A Cognitive View. Springer Netherlands.

Ausubel, D., Novak, J., \& Hanesian, H. (1978). Educational Psychology: A Cognitive View (2nd Ed.). New York: Holt, Rinehart \& Winston.

Bosse, Y. e Gerosa, M. A. (2015) Reprovações e trancamentos nas disciplinas de introdução à programação da universidade de São Paulo: um estudo preliminar. In Workshop sobre Educação em Informática, Recife, Julho de 2015.

Ezenwoye, O. (2018) What language? - The choice of an introductory programming language. In 2018 IEEE Frontiers in Education Conference (FIE). IEEE, 2018.

Gu, Lingkang \& Yan, Nan \& Xiu, Yu. (2017). Discussion on Teaching Methods and Choice of Programming Language on Software Engineering Major. DEStech Transactions on Engineering and Technology Research. 
Guo, P. J. (2018). Non-native english speakers learning computer programming: Barriers, desires, and design opportunities. In Proceedings of the 2018 CHI Conference on Human Factors in Computing Systems, pages 1-14.

Karatrantou, A., Panagiotakopoulos, C. e Patras, A. (2008). Algorithm, pseudo-code and lego mindstorms programming. In Proceedings of International Conference on Si- mulation and Programming for Autonomous Robots/Teaching with Robotics: Didactic Approaches and Experiences, Venice, Italy, pages 70-79.

Klir, J. e Yuan, B. (1995) Fuzzy sets and fuzzy Logic - theory and applications. Upper Saddle River: Prentice-Hall PTR.

Olatunji, E. K., Oladosu, J. B., Odejobi, O. A. e Olabiyisi, S. O. (2019). A needs assessment for indigenous african language-based programming languages. Annals of Science and Technology, 4(2):1-5.

Oliveira, O. L., Monteiro, A. M. e Roman, N. T. (2013). Can natural language be utilized in the learning of programming fundamentals? In 2013 IEEE Frontiers in Education Conference (FIE), pages 1851-1856. IEEE.

Olsen, A. L. (2005). Using pseudocode to teach problem solving. Journal of Computing Sciences in Colleges, 21(2), 231-236.

Rissoli, V., Giraffa, L., e Barone, D. (2007). Uma Proposta de Aplicação de Lógica Fuzzy para Modelagem do Processo de Raciocínio de um Assistente Virtual. Brazilian Symposium on Computers in Education (Simpósio Brasileiro de Informática na Educação - SBIE), 1(1), 350-359.

Watkins, D., Marsh, H. W. e Young, D. (1987). Evaluating tertiary teaching: A new zealand perspective. Teaching and Teacher Education, 3(1): 41-53.

Watson, C. e Li, F. W. (2014). Failure rates in introductory programming revisited. In Proceedings of the 2014 conference on Innovation \& technology in computer science education, pages 39-44. 